\title{
The influence of height and key on the perceptual similarity of transposed melodies
}

\author{
RENÉ VAN EGMOND, DIRK-JAN POVEL, and ERIC MARIS \\ University of Nijmegen, Nijmegen, The Netherlands
}

\begin{abstract}
In two experiments, the perceptual similarity between a strong tonal melody and various transpositions was investigated using a paradigm in which listeners compared the perceptual similarity of a melody and its transposition with that of the same melody and another transposition. The paradigm has the advantage that it provides a direct judgment regarding the similarity of transposed melodies. The experimental results indicate that the perceptual similarity of a strong tonal melody and its transposition is mainly determined by two factors: (1) the distance on the height dimension between the original melody and its transposition ( pitch distance), and (2) the distance between keys as inferred from the circle of fifths (key distance). The major part of the variance is explained by the factor pitch distance, whereas key distance explains only a small part.
\end{abstract}

Transposition is a frequently used transformation on melodies in musical compositions. In an exact transposition, each tone of a melody is shifted by the same number of semitones on the height dimension. The height dimension reflects the logarithmic musical scale that listeners use to represent melodies (Attneave \& Olson, 1971; Shepard, 1982). The magnitude of the shift on the height dimension is called pitch distance. A transposition applied to a tonal melody (a melody that induces a key) entails a key change, except in the case of an octave transposition. The magnitude indicating the relationship between keys is called key distance (see, e.g., Takeuchi \& Hulse, 1992). Pitch distance and key distance figure as the main variables in this study in which similarity judgments of transposed melodies are investigated.

\section{Relation Between Key Distance and Pitch Distance in Transposed Melodies}

In Figure 1, the 12 major keys (indicated by their most common names) are presented on the circle of fifths. In this figure, there are two sets of numbers adjacent to the key names: (1) outside the circle - for each key, its key distance (in steps on the circle of fifths) relative to the key of $\mathrm{C}$ major; (2) inside the circle - for each key, the minimal pitch distances (in semitones), corresponding to the downward and upward transpositions, respectively, notated on the two sides of the slash, from $\mathrm{C}$ major to that key. For example, the transposition of a melody from $\mathrm{C}$ major to $\mathrm{G}$ major can be realized with the Pitch Distances 5 and 7 , respectively, a downward transposition $\left(\mathrm{C}_{3} \rightarrow \mathrm{G}_{2}\right)$ and an upward transposition $\left(\mathrm{C}_{3} \rightarrow \mathrm{G}_{3}\right)$.

The authors are grateful to David Butler, Stephen Handel, Stewart Hulse, and two anonymous reviewers for the helpful suggestions made on an earlier version of this paper. Correspondence should be sent to R. van Egmond, Nijmegen Institute for Cognition and Information, University of Nijmegen, P.O. Box 9104, 6500 HE Nijmegen, The Netherlands (e-mail: egmond@nici.kun.nl).
Three important properties can be derived from this figure. First, a step clockwise or counterclockwise on the circle of fifths results in the same key distance, so that only six different key distances are possible. Second, within the range of an octave a transposition to a specific key can be realized by two different pitch distances (e.g., $\mathrm{C}_{3} \rightarrow \mathrm{G}_{2}, \mathrm{C}_{3} \rightarrow \mathrm{G}_{3}$ ), indicating that key distance and pitch distance are, to some extent, related and cannot be varied entirely independently. Third, for a given pitch distance (e.g., five semitones), transpositions to two different keys that are situated on opposite sides of the original key (in Figure 1, the key of $\mathrm{C}$ major) are possible (in the present example, $\mathrm{C} \rightarrow \mathrm{G}$ and $\mathrm{C} \rightarrow \mathrm{F}$ ), both transpositions involving the same key distance. In conclusion, the individual contribution of the variables pitch distance and key distance can be properly investigated only when a given key distance is combined with different degrees of pitch distance.

\section{Similarity Judgments on Transposed Tonal Melodies}

Although pitch distance is the basic characteristic of transpositions of both tonal and nontonal melodies, its influence and, if applicable, its interaction with key distance has hardly been investigated systematically. However, a few studies have reported that similarity judgments between a melody and its transposition are greatly influenced by the pitch distance between a melody and its transposition, and not by key distance (Francès, 1958/ 1988, p. 177; Hershman, 1994; van Egmond \& Povel, 1994b, 1996).

With regard to key distance, several studies reported that transpositions to near keys (keys at nearby positions on the circle of fifths) are perceived as more similar to the original melody than are far-key transpositions (e.g., Cuddy, Cohen, \& Mewhort, 1981; Cuddy, Cohen, \& Miller, 1979; Takeuchi \& Hulse, 1992; Trainor \& Trehub, 1993). In addition, part of the results obtained in research 


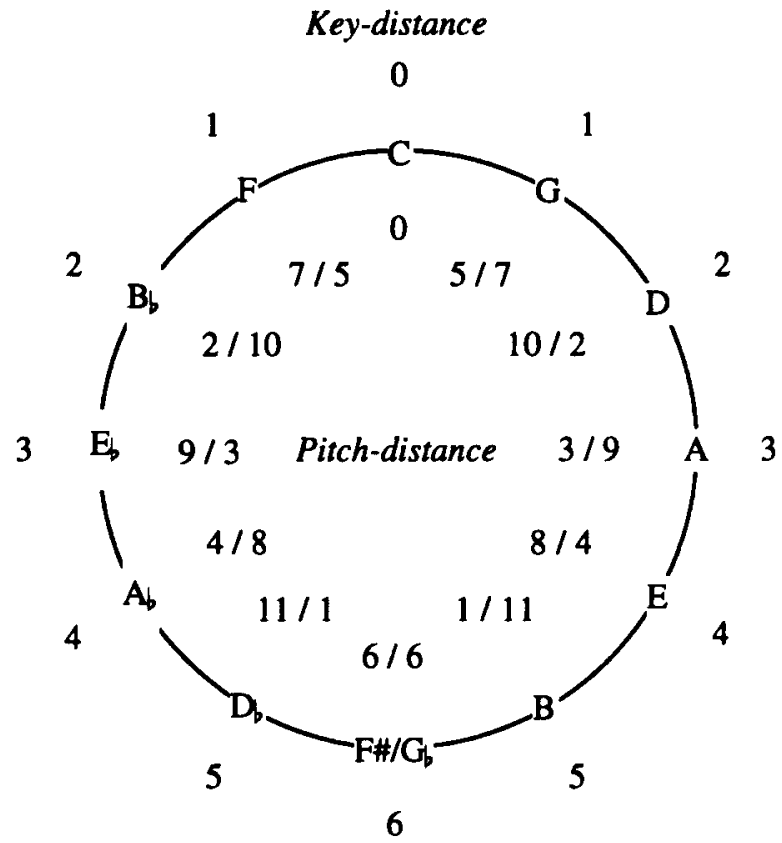

Figure 1. The circle of fifths representing the 12 major keys. On the outside of the circle, key distance relative to the key of C major is presented. Inside the circle, for each key, two pitch distances left and right of the slash are presented corresponding to a downward pitch shift and an upward shift, respectively, from C major to that key.

on transposition is obscured by two confounding factors, discussed below.

One factor concerns the fact that key distance effects depend on how strongly a melody actually induces a key in the listener. One might assume logically that key distance effects would depend on how strongly a melody actually induces a key in the listener. Several characteristics in melodic sequences have been shown to play a role in key induction: (1) the presence of the tones of a diatonic scale (Bartlett \& Dowling, 1980; Dowling, 1978, 1991; Krum- hansl, 1990), (2) the presence of rare intervals, such as the tritone (Brown \& Butler, 1981; Butler, 1983, 1989), (3) the order of the tones (Bartlett \& Dowling, 1980; Bharucha, 1984b; Brown, Butler, \& Jones, 1994; Deutsch, 1984), and (4) the underlying harmony (Croonen, 1991; Croonen \& Kop, 1989; Cuddy et al., 1981).

A second factor is related to the possibility that a transposition may be perceived as a shift on the scale of the original melody, which will be referred to as a tonal transposition. A tonal transposition may either be exact (same interval structure) or inexact (changed interval structure). For example, if the sequence C-D-E (in the key of C) is shifted to $\mathrm{G}-\mathrm{A}-\mathrm{B}$, this transposition-having the same interval structure as the original melody - may be perceived as a tonal transposition within the original key of $\mathrm{C}$ instead of a transposition involving a key change to $\mathrm{G}$. However, if the sequence $\mathrm{C}-\mathrm{D}-\mathrm{E}$ is shifted to $\mathrm{E}-\mathrm{F}-\mathrm{G}$, the interval structure changes, resulting in an inexact tonal transposition. Both exact and inexact tonal transpositions are perceptually similar to the original melody (Bartlett \& Dowling, 1980; Dewitt \& Crowder, 1986; Dowling, 1978, 1986; van Egmond \& Povel, 1996). In some cases, tonal transpositions, which do not yield a change in key, have been used to provide evidence concerning key-distance effects (Takeuchi \& Hulse, 1992; van Egmond \& Povel, 1994a).

To avoid the discussed interactions, we used in this study only strong tonal melodies and transpositions that cannot be interpreted as tonal transpositions. The perceptual similarity between a strong tonal melodic sequence and various transpositions is studied by systematically varying the factors pitch distance and key distance. To study this perceptual similarity we have chosen a similaritycomparison paradigm, in which subjects compared two consecutive combinations of two melodies and indicated in which combination the melodies were more similar (see Figure 2). As shown in the figure, the combinations comprise the same standard melody but different transpositions, while both combinations are preceded by the same

Pair
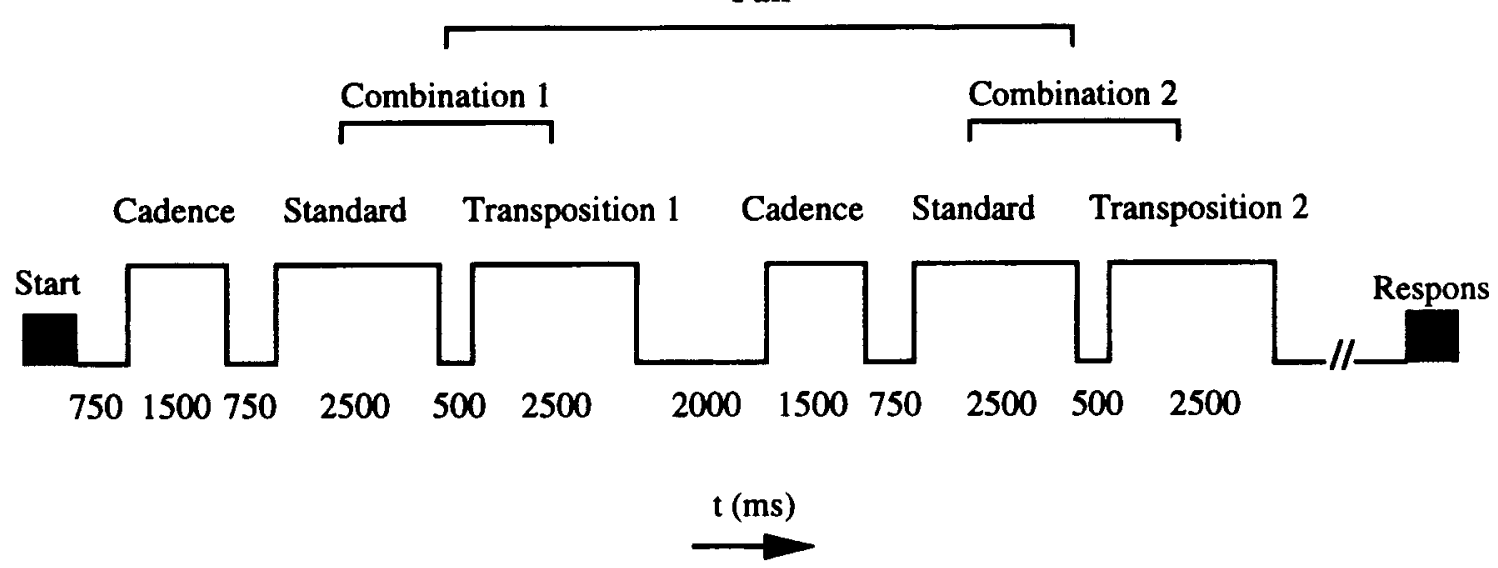

Figure 2. Display of the paradigm used in the experiments. A pair consists of two combinations that contain the same standard melody but different transpositions (comparison melodies). Standard melodies are preceded by a V-I cadence. Numbers indicate the duration of the different parts and pauses in a trial (in milliseconds). 
cadence to induce the key of the standard melody. A pair thus consists of two uniquely coupled combinations.

We believe that this paradigm, which has not been used before for studying transpositions, has a distinct advantage over the standard-comparison paradigm often used in transposition studies (Bartlett \& Dowling, 1980; Cuddy et al., 1979; Dewitt \& Crowder, 1986; Dowling, 1978; Francès, 1958/1988, p. 177; Hershman, 1994; Takeuchi \& Hulse, 1992; Trainor \& Trehub, 1993; van Egmond \& Povel, 1994b). In the latter paradigm (in which the comparison melody may be either transposed or not), a subject indicates whether the standard and comparison melodies are identical. To make the task not completely trivial, part of the comparison melodies (often $50 \%$ ) is altered by substituting one tone for another (the "lures"). As a result, this paradigm is only able to indicate how transpositions interfere with the subject's judgment of the structural identity of the melodies - that is, it provides only indirect information regarding the effect of transposition on the perceived similarity. Moreover, the introduction of an altered tone introduces an additional variable that may strongly influence a listener's judgment, depending on the position of the altered tone in the melody and the type of (diatonic or chromatic) alteration (see, e.g., Bharucha, 1984a; Trainor \& Trehub, 1994; van Egmond \& Povel, 1996). In contrast, the similarity comparison paradigm used in this study does not have to introduce lures (and, consequently, an additional variable) but obtains a direct estimate of the similarity between different transpositions that are created by manipulating the variable(s) under investigation.

\section{EXPERIMENT 1}

The perceptual similarity between a strong tonal melody and various transpositions was studied. Transposition factors pitch distance and key distance were varied independently, as far as possible, by combining three pitch distances with three key distances.

\section{Method}

\section{Subjects}

Twenty subjects - graduate and undergraduate students of the University of Nijmegen-participated in the experiment. Fifteen subjects received course credits, and 5 were volunteers. The median age of the subjects was 24 years. None of the subjects were professional musicians, and none had received formal musical ear training. All of them had played or were playing an instrument for several years, with an average of 6 years.

\section{Apparatus}

Stimulus presentation and response collection were managed by a special program written on an Atari 1040 STf computer. The sounds were presented using a Roland Rhodes 760 synthesizer controlled by the Atari computer through MIDI (Musical Instrument Digital Interface). The melodies were presented using a harpsichord sound (Harpsichord 3 ) and the cadence using a piano sound (Acoustical Piano 1). The different instruments were used to facilitate the distinction between melody and cadence. The relative loudness of the two instruments was left to the standard setting of the de- vice. The stimuli were presented through a loudspeaker (Kawai, KM20) at a comfortable listening level that could be adjusted by the subjects.

\section{Stimuli}

Cadence and standard melody. The cadence preceding the standard melody consisted of a V-I chord progression constructed using standard voice-leading rules (see, e.g., Aldwell \& Schachter, 1989). Because the goal was to study how perceived similarity varied with the transposition factors pitch distance and key distance, it was decided to keep the structure of the original melody constant - that is, to use only one standard melody. The standard melody (see Figure 3) had the following characteristics: (1) all major diatonic scale tones occurred in it, and (2) it had a strong tonal structure.

The first characteristic guarantees that, irrespective of the key to which the melody is transposed, all elements of the new key are present. The second characteristic was added to enhance key induction, as discussed in the introduction. The melody used can be conceived of as a sequence of broken chords (I-II-V-I) and is based on the characteristics of strong tonal melodies, as described by Cuddy et al. (1981).

Comparison melodies. The transpositions (comparison melodies) were constructed by combining three pitch distances with three key distances in the following way. A (default) upward transposition to the positions 1,3 , and 5 steps clockwise on the circle of fifths (e.g., from the key of $\mathrm{C}$ to $\mathrm{G}, \mathrm{A}$, and $\mathrm{B}$, respectively) resulted in three pitch distances: 7,9 , and 11 , respectively. Two additional pitch distances for each key distance were obtained by shifting the default transposition one octave up and one octave down. Figure 4 shows a complete description of the used pitch distances and key distances. Each transposition is thus characterized by a given change in key (factor key distance) and by a given shift in height (factor pitch distance).

Pairs of standard and comparison melodies. Each standard melody plus a transposition (Combination 1 in Figure 2) was combined with the same standard melody and all other transpositions (Combination 2 in Figure 2). With these nine stimuli, combination of a standard and a transposition, 36 pairs were formed. Each of these pairs was presented twice in two successive sessions; thus, the subjects received 72 trials. The order of presentation of the trials and the order of the two combinations within a trial were randomized per subject.

\section{Procedure}

The subject's task was to indicate in which combination he/she perceived the transposition as more similar to the standard (sec Figure 2). After each trial, the subject could choose either to answer immediately or to repeat the trial (the number of repetitions was left to the subject). Before starting the experiment proper, the subjects were familiarized with the procedure using six practice trials. No feedback was given during these trials. The subjects answered by clicking the mouse on buttons appearing on the screen.

In each trial, the key for cadence and standard melody was randomly selected out of 12 possible keys, with the first tone of the standard melody ranging from $\mathrm{G}_{4}$ to $\mathrm{F}_{5}$. The lowest tone (tonic) of the cadence was always two octaves below the lowest tone of the

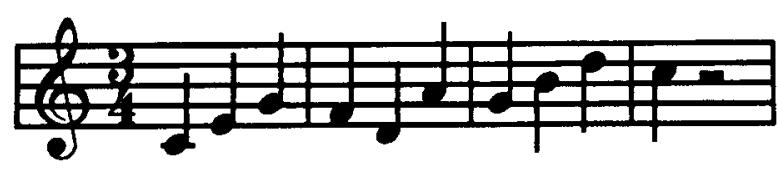

Figure 3. The standard melody (presented in the key of $\mathrm{C}$ major) used in Experiments 1 and 2. The melody is based on the chordal progression I-II-V-I. 


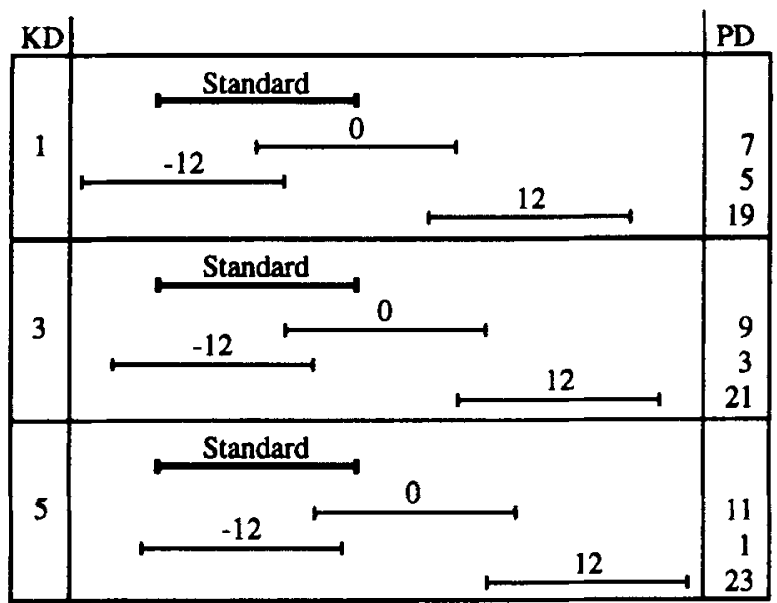

Figure 4. A graphical display of the transpositions used in Experiment 1 . The default direction is indicated with a 0 ; the other two transpositions are indicated with -12 and +12 , respectively, indicating the downward and upward octave shift. For each transposition, key distance (KD) and pitch distance (PD) are given.

standard. The key of the comparison melody was, of course, varied relative to the selected key for the standard melody.

Timing and order of cadences, standard, and comparison melodies are shown in Figure 2. The timing was determined in such a way that no conflict could occur with the (implied) meter of the original melody (see Figure 1). A trial began 750 msec after a start button was pressed. Only the interonset interval (IOI) of the tones was controlled; the decay in tone amplitude during the IOI was left to the default setting of the synthesizer. The tones of the cadences had an IOI of $750 \mathrm{msec}$. Between cadence and standard was a pause of $750 \mathrm{msec}$. All tones of standard and comparison melodies had an IOI of $250 \mathrm{msec}$. Between standard and comparison melodies was a pause of $500 \mathrm{msec}$, and between Comparison Melody 1 and the next cadence was a pause of $2,000 \mathrm{msec}$.

\section{Method of Analysis}

Consistency percentage. For each pair (combinations of standard and transposition melodies), a preference score was obtained on the basis of the individual judgments (preferences). Because each pair was judged twice, a consistency measure could be derived for each subject, indicating the percentage of pairs that were judged the same when offered the second time. Thus, if a subject scored $100 \%$ consistency, he/she gave exactly the same answer to all the pairs. Those subjects that had a consistency score equal to or lower than $50 \%$ were discarded from further analysis.

Analysis of preference scores using the Bradley-Terry-Luce model. To analyze the preference scores, we used the model developed by Bradley and Terry (1952) and Luce (1959), which we will refer to as the $B T L$ model. The BTL model is a variant of the model of comparative judgment of Thurstone (1927) and amounts to a onedimensional Thurstonian analysis with a logistic distribution. The BTL model yields a scale value $\left(b_{i}\right)$ for each stimulus estimated by means of the method of least squares. To explain the application of the BTL model in the analysis of preference scores, we use an example of three stimuli presented in Table 1 . The table comprises different subtables (matrices), in which rows are indicated with $i$ and columns with $j$.

The cells in the first matrix in Table 1 present the number of times $\left(F_{i j}\right)$ stimulus $i$ is preferred over stimulus $j$ for 100 hypothetical subjects. In the second matrix, the "observed" preference proportions $\left(p_{i j}\right)$ are derived by dividing $F_{i j}$ by the number of measurements per pair $(N=100)$, resulting in a number between 0 and 1 . This proportion corresponds to the chance that stimulus $i$ will be preferred over $j$. For $i=j$, it is assumed that $p_{i j}=.50$.

The BTL model assumes the following relation between $p_{i j}$ and the difference between the scale values $\left(b_{i}-b_{j}\right)$ to be obtained:

$$
p_{i j}=\frac{1}{1+e^{-\left(b_{i}-b_{j}\right)}} \text {. }
$$

In the BTL model, the difference between the scale values $\left(b_{i}-b_{j}\right)$, also indicated as $B_{i j}$, is computed using the inverse of Equation 1:

$$
B_{i j}=b_{i}-b_{j}=\ln \frac{p_{i j}}{1-p_{i j}} .
$$

The differences between the scale values $\left(B_{i j}\right)$ are presented in the third matrix of Table 1 . The scale values $\left(b_{i}\right)$ are then derived by summing rows and dividing this sum by the number of stimuli. For every stimulus, this results in $\bar{B}_{i}=b_{i}-\bar{b}$, in which $b_{i}$ is the scale value of stimulus $i$, and $\bar{b}$ is the mean of all scale values. The mean of the row, $\bar{B}_{i,}$, thus equals the scale value of stimulus $i$ in terms of its deviation from the mean of all scale values. Because $\bar{b}$ is a constant and scale values are unique up to a translation, $\bar{b}$ can be taken to be zero, and, thus, the mean of the rows equals the scale values.

To determine the tenability of the model, a matrix of predicted values $\left(b_{i}-b_{j}\right)$ is composed by subtracting all scale values from each other. Using Equation 1, the predicted values $\left(b_{i}-b_{j}\right)$ are then transformed into predicted preference proportions $\left(\pi_{i j}\right)$. The observed and predicted proportions are used in a chi-square goodness-of-fit test [in this case, yielding $\chi^{2}(1)=0.55, p=.46$ ]. A nonsignificant value of chi-square means that the observed and predicted proportions do not differ significantly and, consequently, indicates that the use of the BTL model is appropriate.

\section{Results}

Fourteen subjects scored a consistency higher than $50 \%$, and they were included in the final analysis. The preference scores of these subjects were analyzed using the BTL model, resulting in scale values for each transposition. A chi-square goodness-of-fit test shows that the BTL model for these data cannot be rejected $\left[\chi^{2}(28)=\right.$ $29.4, p=.39]$.

Table 1

\begin{tabular}{|c|c|c|c|c|c|c|c|c|c|c|c|c|c|c|c|c|c|}
\hline \multirow[b]{3}{*}{ Stimuli } & & & & \multicolumn{6}{|c|}{ Observed } & & & \multicolumn{6}{|c|}{ Predicted } \\
\hline & \multicolumn{3}{|c|}{$F_{i j}$} & \multicolumn{3}{|c|}{$p_{i j}$} & \multicolumn{3}{|c|}{$b_{i}-b_{j}$} & \multicolumn{2}{|c|}{ Scale Values } & \multicolumn{3}{|c|}{$b_{i}-b_{j}$} & \multicolumn{3}{|c|}{$\pi_{i j}$} \\
\hline & 1 & 2 & 3 & 1 & 2 & 3 & 1 & 2 & 3 & Sum & $b_{t}$ & 1 & 2 & 3 & 1 & 2 & 3 \\
\hline 1 & & 80 & 90 & .50 & .80 & .90 & 0 & 1.39 & 2.20 & 3.59 & 1.20 & 0 & 1.49 & 2.09 & .50 & .82 & .89 \\
\hline 2 & 20 & & 62 & .20 & .50 & .62 & -1.39 & 0 & 0.49 & -0.90 & -0.30 & -1.49 & 0 & 0.60 & .18 & .50 & .65 \\
\hline 3 & 10 & 38 & & .10 & .38 & .50 & -2.20 & -0.49 & 0 & -2.69 & -0.90 & -2.09 & -0.60 & 0 & .11 & .35 & .50 \\
\hline
\end{tabular}

Example of the Use of the BTL Model With Three Stimuli (1, 2, and 3) and for 100 Hypothetical Subjects

Note-The table contains several subtables (matrices) describing the relation between stimuli $i$ and $j . F_{i j}$, the number of times stimulus $i$ was preferred over stimulus $j ; p_{i j}$, the observed preference proportion; $b_{i}-b_{j}$, the difference between the scale values; $b_{i}$, the scale values; $\pi_{i j}$, the predicted preference proportion. 


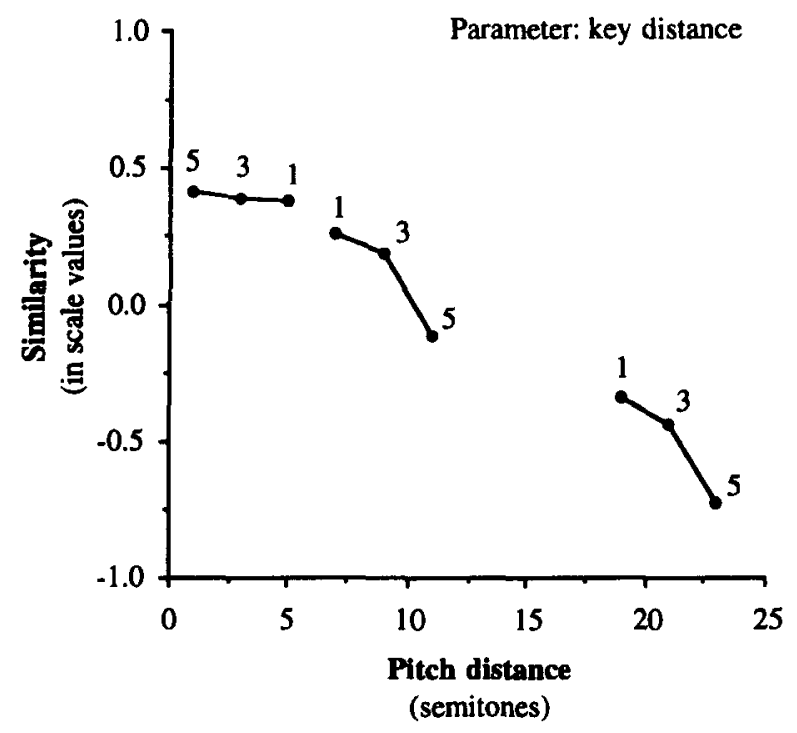

Figure 5. Similarity measures (scale values) obtained in Experiment 1 . Similarity is plotted against pitch distance. The key distance values of the stimuli are printed near the data points. The connection lines are added to improve readability only.

Figure 5 shows for each transposition the perceived similarity (in scale values) as a function of pitch distance. The graph shows a strong relation between similarity and pitch distance-similarity decreasing with increasing pitch distance. The key-distance values of the transpositions are indicated near the data points in the graph. Comparing the similarity scores for the three key distances, a trend is found to judge transpositions to a near key (Key Distance 1) as more similar than those to a far key (Key Distance 5), with Key Distance 3 taking an intermediate position. However, the difference between Key Distances 3 and 5 is larger than the difference between Key Distances 1 and 3 for Pitch Distances 9 and 21 .

Pitch distance and key distance are practically uncorrelated $(r=.0711)$. The correlation between pitch distance and similarity is $-.977(p=.0001)$, and the correlation between key distance and similarity is -.255 $(p=.51)$. Pitch distance is the first variable used in the regression analysis, because this factor has the strongest relation (the highest correlation) with similarity.

Multiple regression performed on the scale values, taking into account the independent variables pitch distance and key distance, confirms the relations inferred from the graph. The results of the multiple regression analysis show that the total amount of explained variance is $98.9 \%[F(2,6)=261, p=.0001]$, of which $95.4 \%$ $[F(1,6)=487, p \ll .001]$ is attributable to pitch distance and $3.36 \%$ [given by the semipartial coefficient of determination, $F(1,6)=17.8, p<.01]$ is attributable to key distance. The estimated regression function is

$$
\begin{aligned}
\text { similarity }= & .69+(-.05 \text { pitch distance } \\
& -.045 \text { key distance }) .
\end{aligned}
$$

The coefficients of both pitch distance and key distance are negative, indicating that an increase in pitch distance or key distance results in a decrease of similarity.

\section{Discussion}

The presented analysis has shown that both factors significantly affect the perceived similarity of standard and transposed melodies, although the contribution of the factor pitch distance is far greater than that of the factor key distance. Pitch distance explains $95.4 \%$ of the variance; key distance explains only $3.36 \%$. These findings confirm earlier studies that also reported a major influence of pitch distance on the perceived similarity, when this factor and key distance are varied (see Francès, 1958/ 1988, p. 177; Hershman, 1994; van Egmond \& Povel, $1994 \mathrm{~b}$ ). The findings also indicate that when a melody is transposed to a specific key (e.g., $C \rightarrow G$ ), perceived similarity is greatly dependent on whether the transposition is upward $\left(\mathrm{C}_{3} \rightarrow \mathrm{G}_{3}\right)$ or downward $\left(\mathrm{C}_{3} \rightarrow \mathrm{G}_{2}\right)$. Although key distance is only a minor factor in this study, its effect is in the expected direction: an increase in key distance is accompanied by a decrease in perceived similarity.

Because, in Experiment 1, the factor pitch distance was varied at nine different levels and the factor key distance at only three different levels, it might be argued that the listener's judgment has mainly been influenced by the more salient factor pitch distance. To obtain a more balanced view of the importance of the factor key distance, in Experiment 2, we increased the number of levels of the variable key distance while keeping the variation of pitch distance minimal.

\section{EXPERIMENT 2}

Experiment 2 used the same paradigm and the same standard melody as were used in Experiment 1, but different transpositions were included. The transposition factors key distance and pitch distance were each varied at five levels, and each key distance was selected at two different positions on the circle of fifths: one clockwise (e.g., $\mathrm{C} \rightarrow \mathrm{G}$ ) and one counterclockwise (e.g., $\mathrm{C} \rightarrow \mathrm{F}$ )that is, transpositions were made to both sharp and flat keys. Furthermore, pitch distance was restricted in such a manner that, for each key distance, the minimum possible pitch distance was obtained. This minimized the effect of pitch distance on key distance, enabling an unbiased assessment of the key distance effect.

\section{Method}

\section{Subjects}

Twenty-six subjects-graduate and undergraduate students of the University of Nijmegen--participated in the experiment. The 26 subjects received payment for their participation (an amount in Dutch guilders equivalent to US \$5). The median age of the subjects was 24 years. None of the subjects were professional musicians, and none had received formal musical ear training. All of them had played or were playing an instrument for several years, with an average of 6.5 years. 


\section{Stimuli}

The standard melody was the same as that in Experiment 1. Five values for key distance were used $(1,2,3,4$, and 5 steps on the circle of fifths), each selected at two positions on the circle of fifths (clockwise and counterclockwise), resulting in 10 transpositions. The values of pitch distance were determined in such a way that the variation over key distances was minimized while the size was minimal for each key distance, thus limiting the possible influence of this factor on the similarity judgments. The standard melody and the 10 resulting transpositions are shown in Figure 6.

With the 10 stimuli, combination of a standard and a transposition, 45 pairs were formed. As each pair was presented twice in two successive sessions, subjects received 90 trials.

\section{Apparatus and Procedure}

The apparatus and procedure used in Experiment 1 were also used in Experiment 2.

\section{Results}

Twenty-one subjects scored a percentage consistency higher than $50 \%$, and they were included in the final analysis. The preference scores of these subjects were analyzed using the BTL model, resulting in scale values for each transposition. A chi-square goodness-of-fit test shows that the BTL model for these data cannot be rejected $\left[\chi^{2}(36)=\right.$ $35.0, p=.51]$.

Similarity (in scale values) is shown as a function of pitch distance in Figure 7 for each transposition. We observe the same relation between similarity and pitch dis-

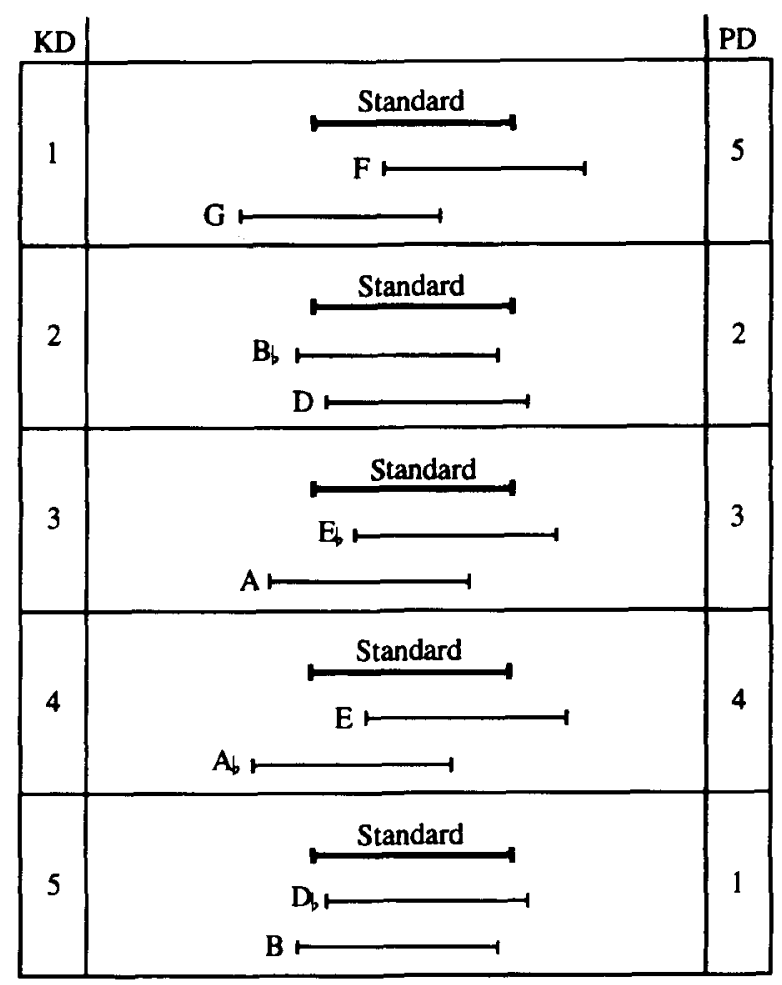

Figure 6. A graphical display of the transpositions used in Experiment 2. For each transposition, key distance (KD) and pitch distance (PD) are given. Each key distance is associated with two directions on the circle of fifths (here, indicated by key names relative to the key of $C$ ).

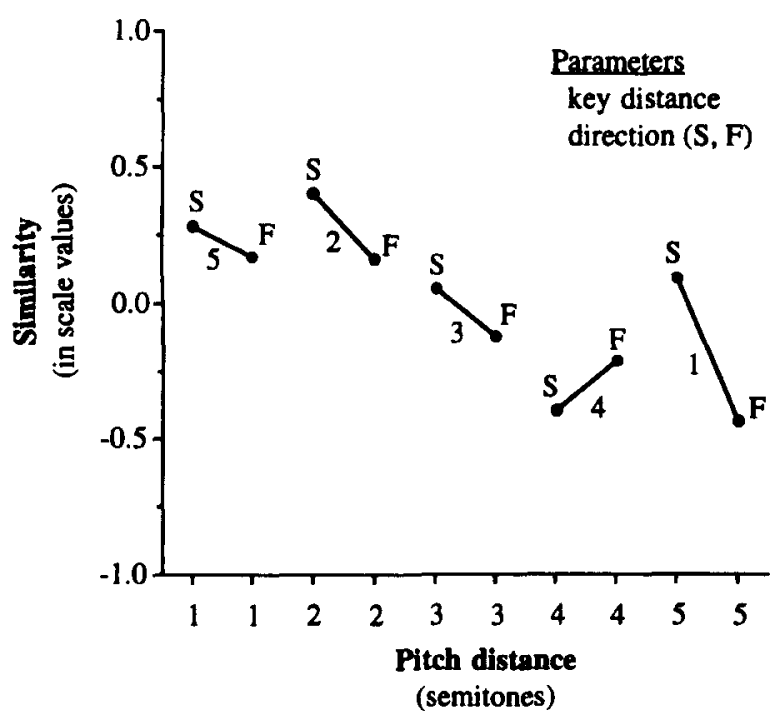

Figure 7. Similarity measures (scale values) obtained in Experiment 2 . Similarity is plotted against pitch distance. The keydistance values of the stimuli are indicated between data points of the same key distance. The direction of the key-distance values on the circle of fifths-clockwise (sharp, S) or counterclockwise (flat, F)-is indicated near the data points. The connection lines are added to improve readability only.

tance as was found in Experiment 1: similarity decreasing with increasing pitch distance. However, the relation is not as clear as in Experiment 1. Key distance does not seem to influence perceived similarity in a systematic way. A trend may be discerned to judge transpositions to sharp keys (clockwise on the circle of fifths) as more similar to the original melody than transpositions to flat keys (counterclockwise on the circle of fifths).

Pitch distance and key distance are intercorrelated to some extent $(r=-.60)$. Since similarity was more highly correlated with pitch distance $(r=-.74)$ than with key distance $(r=.12)$, pitch distance was entered as the first variable in the regression analysis. Because direction is uncorrelated both with pitch distance and key distance, the order of introduction of direction is irrelevant.

Multiple regression performed on the scale values, taking into account the independent variables pitch distance and key distance and the additional factor direction, confirms the relations in the graph. The total amount of explained variance is $80.9 \%[F(3,6)=8.49, p=.01]$ of which $54.1 \%[F(1,6)=21.7, p<.005]$ is attributable to pitch distance, $16.2 \%$ [given by the semipartial coefficient of determination, $F(1,6)=5.11, p<.10$ ] is attributable to key distance, and $10.5 \%[F(1,6)=3.32, p<.15]$ is attributable to direction. The estimated regression function is

$$
\begin{aligned}
\text { similarity }= & .97+(-.20 \text { pitch distance } \\
& -.10 \text { key distance }-.18 \text { direction }) .
\end{aligned}
$$

The coefficients of pitch distance and key distance are negative, indicating that an increase of pitch distance or key distance results in a decrease of similarity. The negative coefficient for direction indicates that transposi- 
tions to sharp keys are perceived as more similar than are transpositions to flat keys.

\section{Discussion}

Analysis of the results of Experiment 2 shows that pitch distance is the only factor that significantly affects the perceived similarity of standard and transposed melodies. The contribution of the factor pitch distance to the explained variance in Experiment 2 was, however, smaller than that in Experiment 1 (54.1\% vs. 95.4\%). This decrease is understandable, since the variation of pitch distance was minimal in Experiment 2.

Although not significant, the contribution of the factor key distance was much higher in Experiment 2 than in Experiment $1(16.2 \%$ vs. 3.6\%). Inspection of Figure 7 reveals no systematic relation between key distance and perceived similarity: only for Key Distances 2, 3, and 4 did we see the expected decrease in similarity, but the similarity measure associated with Key Distance 1 was too low, and that for Key Distance 5 was too high. At present, we do not have an explanation for this phenomenon. But if this finding appears to be replicable, it casts doubt on the assumed inverse relation between key distance (as represented by the circle of fifths) and perceptual similarity.

Also, the third factor varied in Experiment 2, direction (i.e., the selection of sharp or flat keys), appeared not to be significant in the multiple regression analysis. Direction explains $10.5 \%$ of the variance. The graph in Figure 7 shows a trend to judge transpositions to sharp keys as more similar to the original melody than are transpositions to flat keys. This holds for four of the five key distances used; for Key Distance 4, the reverse effect was found. Especially for Key Distance 1, the transposition to the sharp key (e.g., $C \rightarrow G$ ) was greatly preferred over that to the flat key (e.g., $C \rightarrow F$ ). This preference may have been the result of the average listener's adaptation to the common practice in music to modulate to the dominant rather than to the subdominant (Rosen, 1976, p. 33; Schoenberg, 1922/1978, p. 166). A similar effect when transposing to the dominant or subdominant has also been reported in studies by Cuddy and Thompson (1992) and Thompson and Cuddy (1989).

\section{SUMMARY AND CONCLUSIONS}

The combined results of the two experiments may be summarized as follows. Given a tonal melody that strongly induces a key, the similarity between various transpositions and this melody is determined mainly by pitch distance. Key distance and direction on the circle of fifths are less important variables that explain only a small part of the variance. The degree of variance explained by the factor key distance increases slightly when the variation of pitch distance is minimized (as was done in Experiment 2), but it is statistically not significant. This main effect of pitch distance was also confirmed by another study in which the perceived similarity between a (different) melody and the exact and inexact transpositions of that melody was investigated using the similarity paradigm (van Egmond \& Povel, 1996).

Thus, the results presented in this study are in contrast with those of previous studies that reported effects of key distance in the judgment of transposed melodies (e.g., Cuddy et al., 1981; Cuddy et al., 1979; Takeuchi \& Hulse, 1992; Trainor \& Trehub, 1993). Since these previous studies all used a standard-comparison paradigm with lures, one might suppose that the results of the present study were mainly due to the different experimental paradigm used. We do not believe this to be the case, because a number of earlier studies, using a standard-comparison paradigm in which pitch distance was varied, also showed a relatively large effect of pitch distance in judging transpositions (Francès, 1958/1988, p. 177; Hershman, 1994; van Egmond \& Povel, 1994b). In van Egmond and Povel (1994b), a major diatonic scale was transposed to all major keys using upward and downward transpositions. It was found that the perceived similarity was affected by pitch distance, and not by key distance. Furthermore, the results showed that only the size, and not the direction, of the pitch shift affects the recognition. For example, a transposition with a pitch shift -11 (e.g., from C major to $\mathrm{Db}$ major) affected the recognition score to the same extent as did the pitch shift +11 (from $\mathrm{C}$ major to $\mathrm{B}$ major).

As we have argued in the introduction, the variables key distance and pitch distance are, to some extent, interdependent. Thus, in order to obtain insight into the individual contribution of these two variables in the recognition of transpositions, key distance variation must be combined with different levels of pitch distance. Previous studies have usually varied key distance without considering the concomitant pitch distance. Such studies found that key distance is the predominant factor in determining the perceived similarity of transpositions. On the other hand, the results of the present study and those of van Egmond and Povel (1996), in which key distance and pitch distance are systematically varied, indicate that the perceptual similarity of transposed melodies is mainly determined by pitch distance and only to a small extent by key distance. It may be concluded that both factors play a role in the perception of transposed melodies, but their exact interaction is still not quite clear. Therefore, in future research, we plan to study the interaction of the variables pitch distance, key distance, direction on the circle of fifths, and the tonal strength of melodies in more detail using the similarity paradigm employed in this study.

\section{REFERENCES}

Aldwell, E., \& Schachter, C. (1989). Harmony and voice leading (2nd ed.). San Diego, CA: Harcourt Brace Jovanovich.

Attneave, F., \& Olson, R. (1971). Pitch as a medium: A new approach to psychophysical scaling. American Journal of Psychology, 84, 147166.

Bartlett, J. C., \& Dowling, W. J. (1980). Recognition of transposed melodies: A key-distance effect in developmental perspective. Journal of Experimental Psychology: Human Perception \& Performance, 6, 501-515.

Bharucha, J. J. (1984a). Anchoring effects in music: The resolution of dissonance. Cognitive Psychology, 16, 485-518. 
BHARUCHA, J. J. (1984b). Event hierarchies, tonal hierarchies, and assimilation: A reply to Deutsch and Dowling. Journal of Experimental Psychology: General, 113, 421-425.

Bradley, R. A., \& Terri, M. E. (1952). Rank analysis of incomplete block designs: I. The method of paired comparison. Biometrika, 39, 324-335.

Brown, H., \& BUTLER, D. (1981). Diatonic trichords as minimal tonal cue-cells. In Theory Only, 5, 39-55.

Brown, H., Butler, D., \& Jones, M. R. (1994). Musical and temporal influences on key identification. Music Perception, 14, 371-407.

BUTLER, D. (1983). The initial identification of tonal centers in music. In D. R. Rogers \& J. A. Sloboda (Eds.), The acquisition of symbolic skills (pp. 251-262). New York: Plenum.

BUTLER, D. (1989). Describing the perception of tonality in music: A critique of the tonal hierarchy theory and a proposal for a theory of intervallic rivalry. Music Perception, 6, 219-242.

Croonen, W. L. M. (1991). Recognition of tone series containing tonic triads. Music Perception, 9, 231-250.

Croonen, W. L. M., \& KoP, P. F. M. (1989). Tonality, tonal scheme, and contour in delayed recognition of tone sequences. Music Perception, 7, 49-68.

Cuddy, L. L., Cohen, A. J., \& Mewhort, D. J. (1981). Perception of structure in short melodic sequences. Journal of Experimental Psy. chology: Human Perception \& Performance, 7, 869-883.

Cuddy, L. L., Cohen, A. L., \& Miller, J. (1979). Melody recognition: The experimental application of musical rules. Canadian Journal of Psychology, 33, 148-156.

CudDY, L. L., \& ThOMPSON, W. F. (1992). Asymmetry of perceived key movement in chorale sequences: Converging evidence from a probetone analysis. Psychological Research, 54, 51-59.

Deutsch, D. (1984). Two issues concerning tonal hierarchies: Comment on Castellano, Bharucha, and Krumhansl. Journal of Experimental Psychology: General, 113, 413-416.

DewitT, L. A., \& Crowder, R. G. (1986). Recognition of novel melodies after brief delays. Music Perception, 3, 259-274.

DowLING, W. J. (1978). Scale and contour: Two components of a theory of memory for melodies. Psychological Review, 85, 341-345.

Dowling, W. J. (1986). Context effects on melody recognition: Scalestep versus interval representation. Music Perception, 3, 281-296.

Dowling, W. J. (1991). Tonal strength and melody recognition after long and short delays. Perception \& Psychophysics, 50, 305-313.
FRANCÈs, R. (1988). The perception of music (W. J. Dowling, Trans.). Hillsdale, NJ: Erlbaum. (Original work published in 1958)

Hershman, D. P. (1994). Key distance effects in ecological contexts. In 1. DeLiège (Ed.), Proceedings of the 3rd International Conference for Music Perception and Cognition (pp. 243-244). Liège: University of Liège.

KRUMHANSL, C. L. (1990). Cognitive foundations of musical pitch. New York: Oxford University Press.

LuCE, R. D. (1959). Individual choice behavior: A theoretical analysis. New York: Wiley.

Rosen, C. (1976). The classical style (rev. ed.). London: Faber \& Faber. Schoenberg, A. (1978). Theory of harmony (R. E. Carter, Trans.). London: Faber \& Faber. (Original work published in 1922)

SHEPARD, R. N. (1982). Geometrical approximations to the structure of musical pitch. Psychological Review, 89, 305-333.

TAKEUChI, A. H., \& HulSE, S. H. (1992). Key distance effects in melody recognition reexamined. Music Perception, 10, 1-24.

Thompson, W. F., \& CUDDY, L. L. (1989). Sensitivity to key change in chorale sequences: A comparison of single voices and four-voice harmony. Music Perception, 7, 151-168.

Thurstone, L. L. (1927). A law of comparative judgment. Psychological Review, 34, 273-286.

TrainOR, L. J., \& Trehub, S. E. (1993). Musical context effects in infants and adults: Key distance. Journal of Experimental Psychology: Human Perception \& Performance, 19, 615-626.

Trainor, L. J., \& Trehub, S. E. (1994). Key membership and implied harmony in Western tonal music: Developmental perspectives. Perception \& Psychophysics, 56, 125-132.

vaN Egmond, R., \& Povel, D. J. (1994a). Factors in the recognition of transposed melodies: A reply to Takeuchi and Hulse. Music Perception, 12, 137-142.

van Egmond, R., \& Povel, D. J. (1994b). Similarity judgments on transposed melodies as a function of overlap and key-distance. In I. DeLiège (Ed.), Proceedings of the 3rd International Conference for Music Perception and Cognition (pp. 219-220). Liège: University of Liège.

van Egmond, R., \& Povel, D. J. (1996). Perceived similarity of exact and inexact transpositions. Acta Psychologica, 92, 283-295.

(Manuscript received May 9, 1995; revision accepted for publication February 20, 1996.) 